\section{Endoscopic closure of a colonic defect using a novel endoloop system via a single-channel endoscope}

Successful closure of postoperative defects is essential for endoscopic resection of early neoplasms. The endoscopic pursestring suture (EPSS) using a double-channel colonoscope has been shown to be safe and effective. This method involves the interaction of the endoloop and the metal clips through two separate endoscope channels [1]. However, single-channel colonoscopes are more popular in clinical practice because of their longer length, more flexible operation, and cheaper price, especially when used in the right colon. We report on a novel endoloop system that was innovatively applied via a single-channel endoscope.

A 70-year-old man was referred to our center with a 9-month history of increasing defecation. Endoscopic examination with biopsy and histopathology revealed a nongranular type of laterally spreading tumor with moderate atypical hyperplasia in the ileocecal junction. Endoscopic submucosal dissection was performed for en bloc resection. The large defect $(2.5 \times 2.0 \mathrm{~cm})$ was completely closed by EPSS via a single-channel colonoscope (CF Q260; Olympus, Tokyo, Japan), as follows. As an independent device, the nylon loop (Loop-30; LeCamp, Changzhou, China) was first delivered and positioned around the defect. Multiple clips were then applied to fix the endoloop in place. The loop was tightened by grasping the tail with a hook and pulling backward to close the defect ( $\bullet$ Fig. 1, $\bullet$ Video 1 ). The entire procedure was completed via the single channel. The closing process took 8 minutes. The patient was discharged 2 days after the operation without any adverse events.

Lua et al. [2] described their experience of closing a mucosal defect in the stomach using a single-channel gastroscope. To our knowledge, the current case is the

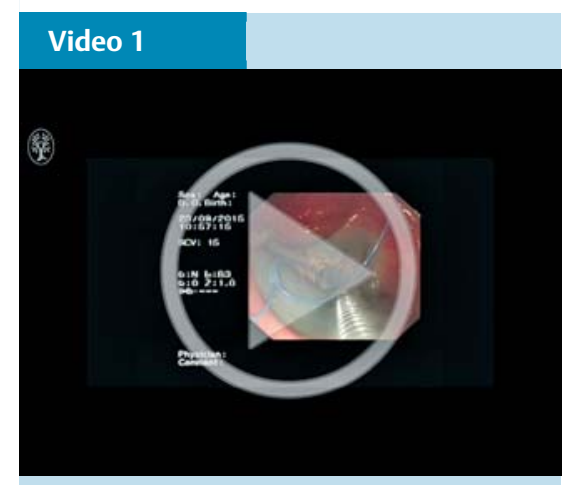

Procedure for closing a colonic mucosal defect using an independent endoloop and clips, via a single-channel colonoscope. The strategy is easy and effective.

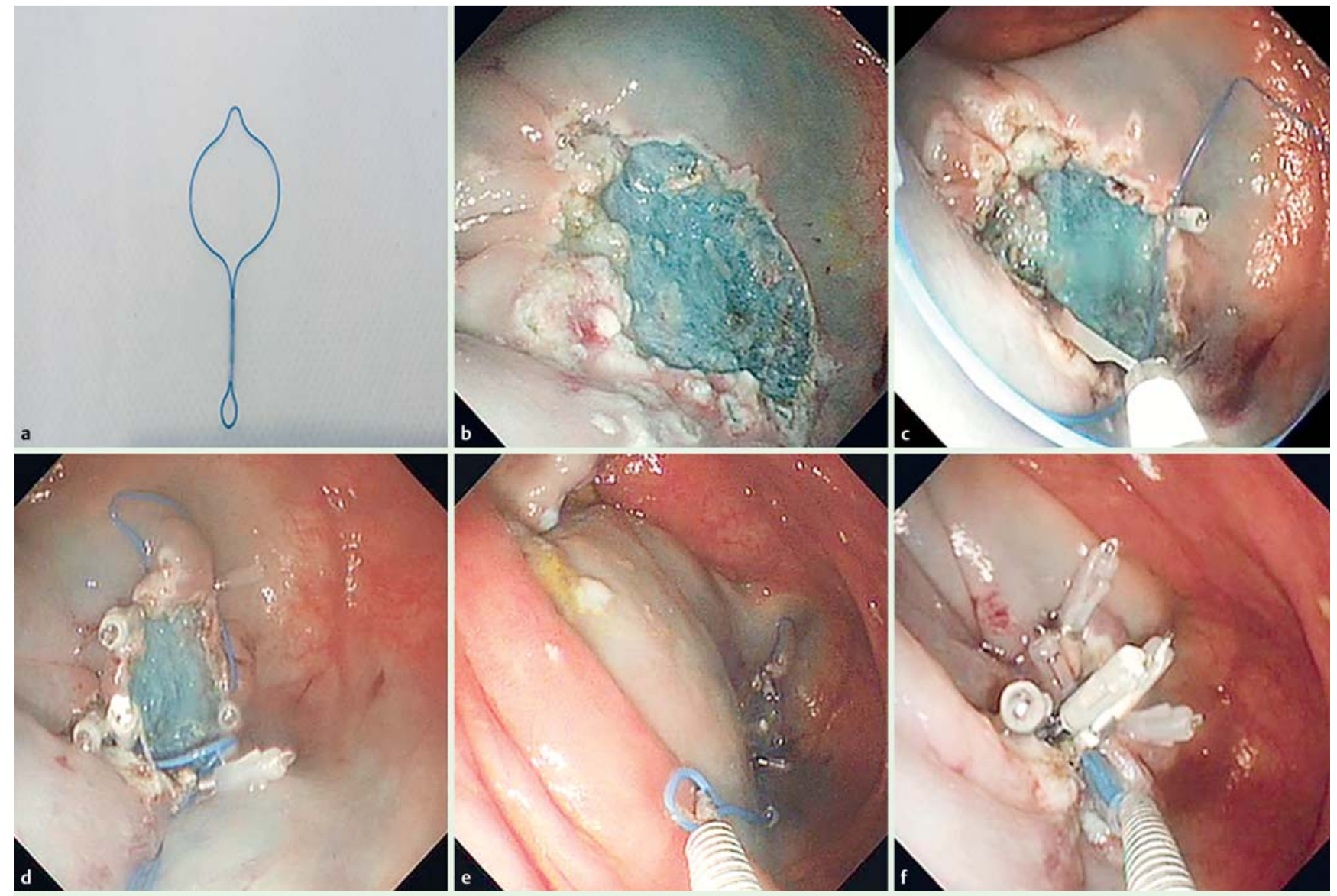

Fig. 1 Closure of a colonic defect using a novel endoloop system via a single-channel colonoscope. a The endoloop. b Endoscopy showed a large colonic defect at the ileocecal junction following endoscopic submucosal dissection. c A separate endoloop was inserted around the mucosal defect and anchored with clips. d Several clips were used to anchor the endoloop.e The endoloop was tightened by grasping the endoloop tail with a hook and pulling backward. f The defect was closed successfully by endoscopic purse-string suture. 
first report on closure of a colonic defect via a single-channel colonoscope. With the assistance of an independent endoloop system, EPSS via a single-channel colonoscope should be feasible, efficient, and safe for endoscopic defect closure.

Endoscopy_UCTN_Code_TTT_1AQ_2AD

\section{Competing interests: None}

\section{Jiankun Wang, Xiang Wang, Li Liu, Lili Zhao, Min Wang, Zhenzhen Liu, Zhining Fan}

Digestive Endoscopy Center, The First Affiliated Hospital of Nanjing Medical University and Jiangsu Province Hospital, Nanjing, China

\section{Acknowledgments}

This study was supported by the National Natural Science Foundation of China (81172266 and 81302107), the Life Health Technology Foundation of Jiangsu province (BL2012031), and the "333 engineering” Foundation of Jiangsu province (BRA2015472).

\section{References}

1 Matsuda T, Fujii T, Emura $F$ et al. Complete closure of a large defect after EMR of a lateral spreading colorectal tumor when using a two-channel colonoscope. Gastrointest Endosc 2004; 60: 836-838

2 Lua GW, Liu F. Closure of large mucosal defect after endoscopic submucosal dissection using "pre-detached loop and clips" method with a single-channel gastroscope. Endoscopy 2015; 47: E464-E465
Bibliography

DOI http://dx.doi.org/

10.1055/s-0042-104192

Endoscopy 2016; 48: E142-E143

(c) Georg Thieme Verlag KG

Stuttgart · New York

ISSN 0013-726X

\section{Corresponding author}

Li Liu, MD and Zhining Fan, MD

Digestive Endoscopy Center

The First Affiliated Hospital of Nanjing Medical University and Jiangsu Province Hospital 300 Guangzhou Road

Nanjing 210029

China

Fax: +86-25-58509931

kit9178@sina.com

fanzhining@njmu.edu.cn 